\title{
Stokes, Maxwell and Darcy: A single finite element approximation for three model problems
}

\author{
Santiago Badia, Ramon Codina* \\ Universitat Politècnica de Catalunya, Jordi Girona 1-3, Edifici C1, 08034 Barcelona, Spain
}

\section{A R T I C L E I N F O}

\section{Article history}

Received 1 July 2010

Received in revised form 29 June 2011

Accepted 2 July 2011

Available online 14 July 2011

\section{Keywords:}

Stabilized finite elements

Compatible approximations

Primal and dual problems

Singular solutions

Nodal interpolations

\begin{abstract}
A B S T R A C T
In this work we propose stabilized finite element methods for Stokes', Maxwell's and Darcy's problems that accommodate any interpolation of velocities and pressures. We briefly review the formulations we have proposed for these three problems independently in a unified manner, stressing the advantages of our approach. In particular, for Darcy's problem we are able to design stabilized methods that yield optimal convergence both for the primal and the dual problems. In the case of Maxwell's problem, the formulation we propose allows one to use continuous finite element interpolations that converge optimally to the continuous solution even if it is non-smooth. Once the formulation is presented for the three model problems independently, we also show how it can be used for a problem that combines all the operators of the independent problems. Stability and convergence is achieved regardless of the fact that any of these operators dominates the others, a feature not possible for the methods of which we are aware.
\end{abstract}

(c) 2011 IMACS. Published by Elsevier B.V. All rights reserved.

\section{Introduction}

The numerical approximation of partial differential equations (PDEs) in general geometries can be performed by using finite element (FE) techniques. The standard approach to the problem consists of considering the weak form of the PDE, and replace the infinite dimensional functional spaces for the solution and test functions by finite dimensional ones. Those finite dimensional spaces are constructed using FE functions over a partition of the domain.

PDEs defined by coercive differential operators can be approximated by the Galerkin FE technique, provided that the corresponding FE space can approximate functions in the continuous functional space; coercivity of the continuous problem is inherited by the discrete one. However, PDEs that exhibit a saddle-point structure, and so stability is attained via a (less demanding) inf-sup condition, cannot be straightforwardly approximated by only looking at the approximability properties of the FE space. The reason is quite simple: inf-sup conditions satisfied by the continuous problem are not inherited (in general) by their discrete versions. Therefore, FE spaces are not only required to exhibit an approximability property, but also a discrete inf-sup condition.

Saddle-point problems include the primal unknown and the dual one, the Lagrange multiplier. FE pairs for these unknowns have to be built such that they satisfy a discrete inf-sup condition (see, e.g., [8]). Examples of linear PDEs with this structure are Stokes' problem, Darcy's problem and Maxwell's problem. Every problem involves a different differential operator, and their well-posedness relies on different inf-sup conditions. It is not surprising that stable FE approximations (called inf-sup stable) are different from one problem to the other. Using inf-sup stable FE methods, e.g. the Stokes problem could be approximated by the Crouzeix-Raviart element [15], Darcy's problem (in dual form) would be solved by using the

\footnotetext{
* Corresponding author.

E-mail addresses: sbadia@cimne.upc.edu (S. Badia), ramon.codina@upc.edu (R. Codina).
} 
every sub-problem separately. We will pay special attention to the design of methods that account for limit values (zero or infinity) of the physical parameters, particularly when dealing with combined problems.

It is observed that the three problems share a saddle point mathematical structure. They all can be written as

$$
\begin{array}{ll}
L_{X}(\boldsymbol{u})+\nabla p=\boldsymbol{f} & \text { in } \Omega, \\
\nabla \cdot \boldsymbol{u}=g & \text { in } \Omega, \\
F_{X}(\boldsymbol{u})=0 & \text { on } \partial \Omega,
\end{array}
$$

where the differential operator $L_{X}$ is given by

$$
L_{X}(\boldsymbol{u})= \begin{cases}L_{S}(\boldsymbol{u}):=-v \Delta \boldsymbol{u} & \text { for the Stokes problem, } \\ L_{M}(\boldsymbol{u}):=\lambda \nabla \times \nabla \times \boldsymbol{u} & \text { for the Maxwell problem, } \\ L_{D}(\boldsymbol{u}):=\sigma \boldsymbol{u} & \text { for the Darcy problem }\end{cases}
$$

and the boundary operator $F_{X}$ is given by

$$
F_{X}(\boldsymbol{u})= \begin{cases}F_{S}(\boldsymbol{u}):=\boldsymbol{u} & \text { for the Stokes problem, } \\ F_{M}(\boldsymbol{u}):=n \times \boldsymbol{u} & \text { for the Maxwell problem, } \\ F_{D}(\boldsymbol{u}):=n \cdot \boldsymbol{u} & \text { for the Darcy problem. }\end{cases}
$$

In the case of Maxwell's problem, $\boldsymbol{f}$ is usually assumed to be divergence free, which together with the boundary condition $p=0$ on $\partial \Omega$ that needs to be added to (3), yields $p=0$ everywhere.

\subsection{Variational form and functional setting}

Let $V_{X} \times Q_{X}$ be the functional spaces where the pair $[\boldsymbol{u}, p]$ is sought for each of the problems introduced earlier. These spaces will be described in what follows in $\Omega$, whenever it makes sense, we get
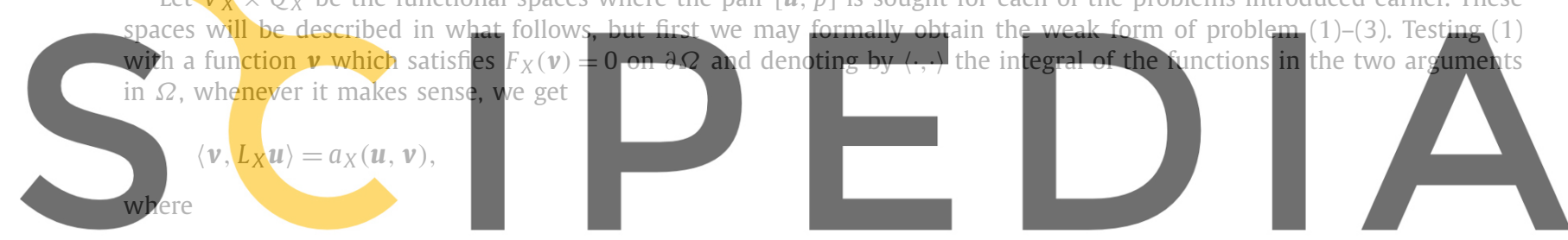

for the Stokes problem,

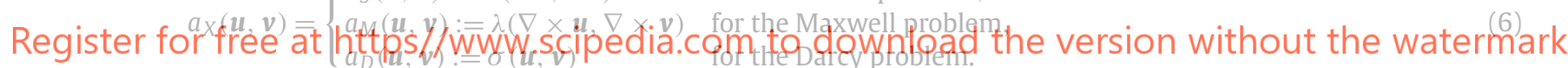

These bilinear forms are bounded (and therefore continuous) if $v \in V_{S}:=H_{0}^{1}(\Omega)^{d}$ in the Stokes case, if $v \in H$ (curl; $\Omega$ ) for the Maxwell problem and if $\boldsymbol{v} \in L^{2}(\Omega)^{d}$ for the Darcy problem. Here and below we will make use of the spaces $H(\operatorname{div} ; \Omega)$ and $H(\mathbf{c u r l} ; \Omega)$, defined as usual as

$$
\begin{aligned}
& H(\operatorname{div} ; \Omega):=\left\{\boldsymbol{v} \in L^{2}(\Omega)^{d} \text { such that } \nabla \cdot \boldsymbol{v} \in L^{2}(\Omega)\right\}, \\
& H(\operatorname{curl} ; \Omega):=\left\{\boldsymbol{v} \in L^{2}(\Omega)^{d} \text { such that } \nabla \times \boldsymbol{v} \in L^{2}(\Omega)^{d}\right\} .
\end{aligned}
$$

A subscript 0 will be added to denote the subspace of functions such that $F_{X}(\boldsymbol{v})=0$ on $\partial \Omega$, with $X$ either $M$ or $D$.

Spaces $V_{X}$ and $Q_{X}$ are determined by requiring that the term $\langle\nabla p, \boldsymbol{v}\rangle$, obtained by testing $\nabla p$ by $\boldsymbol{v}$ is well defined under the minimum regularity conditions. In the Stokes case, since $\boldsymbol{v} \in H_{0}^{1}(\Omega)^{d}$ we may integrate this term by parts and get $\langle\nabla p, \boldsymbol{v}\rangle=-(p, \nabla \cdot \boldsymbol{v})$, so that the space $Q_{s}$ must be $L_{0}^{2}(\Omega)$ (square integrable functions with zero mean over $\Omega$ ).

The situation is different in the case of Maxwell's and Darcy's problem, since in these cases there is an alternative for the choice of $Q_{X}$. Starting with the former, on the one hand, if we do not integrate by parts we have to take $p \in H^{1}(\Omega)$ to guarantee that $\langle\nabla p, \boldsymbol{v}\rangle$ is bounded. Moreover, to ensure that the solution of the problem is $p=0$ for a solenoidal $\boldsymbol{f}$, we may in fact take $p \in H_{0}^{1}(\Omega)$. On the other hand, if we do integrate by parts and take $\langle\nabla p, \boldsymbol{v}\rangle=-(p, \nabla \cdot \boldsymbol{v})$, then we need to require that $p \in L_{0}^{2}(\Omega)$ and $\boldsymbol{v} \in H(\operatorname{div} ; \Omega)$.

For Darcy's problem something similar happens. If we do not integrate by parts we have to take $p \in H^{1}(\Omega)$, and if we do, we need to require that $p \in L^{2}(\Omega)$ and $\boldsymbol{v} \in H(\operatorname{div} ; \Omega)$. The former leads to the so-called primal formulation of Darcy's problem, whereas the latter leads to the dual formulation.

In all cases we need to assume that $\langle\boldsymbol{f}, \boldsymbol{v}\rangle$ is bounded. To simplify a little the discussion we may consider that $\boldsymbol{f} \in$ $L^{2}(\Omega)^{d}$ and $g \in L^{2}(\Omega)$. Likewise, boundary conditions have a different interpretation depending on the choice of the space for $p$ in Maxwell's and Darcy's problems.

The summary of the choices described is presented in Table 1. Note that for the primal formulation of Darcy's problem $\left(V_{X} \times Q_{X}=V_{D 1} \times Q_{D 1}\right)$ the boundary condition $F_{X}(\boldsymbol{u})=0$ is in fact a Neumann-type boundary condition for $p$. 
Table 1

Functional setting for Stokes', Maxwell's and Darcy's problems.

\begin{tabular}{llll}
\hline & $V_{X}$ & $Q_{X}$ & $B_{X}([\boldsymbol{u}, p],[\boldsymbol{v}, q])$ \\
\hline Stokes & $V_{S}=H_{0}^{1}(\Omega)^{d}$ & $Q_{S}=L_{0}^{2}(\Omega)$ & $a_{S}(\boldsymbol{u}, \boldsymbol{v})-(p, \nabla \cdot \boldsymbol{v})+(q, \nabla \cdot \boldsymbol{u})$ \\
Maxwell & $V_{M 1}=H_{0}(\operatorname{curl} ; \Omega)$ & $Q_{M 1}=H_{0}^{1}(\Omega)$ & $a_{M}(\boldsymbol{u}, \boldsymbol{v})+(\nabla p, \boldsymbol{v})-(\nabla q, \boldsymbol{u})$ \\
& $V_{M 2}=V_{M 1} \cap H(\operatorname{div} ; \Omega)$ & $Q_{M 2}=L_{0}^{2}(\Omega)$ & $a_{M}(\boldsymbol{u}, \boldsymbol{v})-(p, \nabla \cdot \boldsymbol{v})+(q, \nabla \cdot \boldsymbol{u})$ \\
\multirow{2}{*}{ Darcy } & $V_{D 1}=L^{2}(\Omega)^{d}$ & $Q_{D 1}=H^{1}(\Omega)$ & $a_{D}(\boldsymbol{u}, \boldsymbol{v})+(\nabla p, \boldsymbol{v})-(\nabla q, \boldsymbol{u})$ \\
& $V_{D 2}=H_{0}(\operatorname{div} ; \Omega)$ & $Q_{D 2}=L_{0}^{2}(\Omega)$ & $a_{D}(\boldsymbol{u}, \boldsymbol{v})-(p, \nabla \cdot \boldsymbol{v})+(q, \nabla \cdot \boldsymbol{u})$ \\
\hline
\end{tabular}

When Eq. (2) is tested by a test function $q \in Q_{X}$ the result has to be treated according to the space $Q_{X}$ chosen. If the result is added to the weak form of (1), the final problem to be solved can be written as follows: find [u, $p] \in V_{X} \times Q_{X}$ such that

$$
B_{X}([\boldsymbol{u}, p],[\boldsymbol{v}, q])=(\boldsymbol{f}, \boldsymbol{v})+(g, q), \quad \forall[\boldsymbol{v}, q] \in V_{X} \times Q_{X},
$$

where the bilinear form $B_{X}$ defined on $\left(V_{X} \times Q_{X}\right) \times\left(V_{X} \times Q_{X}\right)$ is also given in Table 1. As mentioned above, we will take $g=0$ for Maxwell's and Stokes' problems.

Let us discuss now the appropriate working norms. In the Stokes problem, there is no alternative in the choice of the functional spaces. In order to work with a norm that scales correctly and that accounts for the values of the physical parameter $v$, we define

\section{$\|\boldsymbol{v}\|_{V_{s}}:=\|\nabla \boldsymbol{v}\|, \quad\|q\|_{Q_{s}}:=\|q\|$,}

where $\|\cdot\|$ stands for the standard $L^{2}$-norm over $\Omega$. The norm in $V_{S} \times Q_{S}$ is then defined as

$\|[v, q]\|_{S}=v^{\frac{1}{2}}\|v\|_{V_{S}}+v^{-\frac{1}{2}}\|q\|_{Q_{S}}$.

In spite of the fact that all problems discussed are well posed in all functional settings presented (s of this functional framework in the case start considering Maxwell's proble choice $V_{M 2} \times Q_{M 2}$ leads to the curl-div forn curl fprmulation. Find $\boldsymbol{u} \in H_{0}$ (cur

$$
\lambda(\nabla \times \boldsymbol{u}, \nabla \times \boldsymbol{v})+(\nabla p, \boldsymbol{v})=(\boldsymbol{f}, \boldsymbol{v}), \quad \forall v \in H_{0}(\operatorname{curl} ;, \Omega),
$$
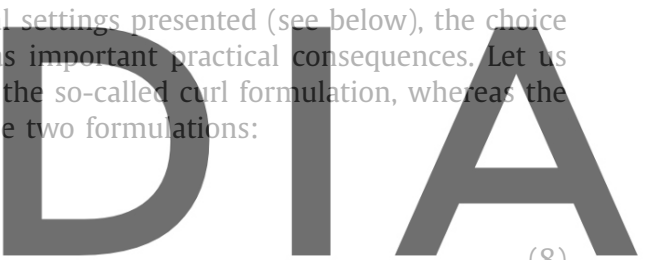

(8) Register for free at https//www.scipedia.com to download the version without the watermark curl-div formulation I. Find $u \in H_{0}(\operatorname{curl} ; \Omega) \cap H(\operatorname{div} ; \Omega)$ and $p \in L_{0}^{2}(\Omega)$ such that

$$
\begin{array}{ll}
\lambda(\nabla \times \boldsymbol{u}, \nabla \times \boldsymbol{v})-(p, \nabla \cdot \boldsymbol{v})=(\boldsymbol{f}, \boldsymbol{v}), & \forall \boldsymbol{v} \in H_{0}(\mathbf{c u r l} ; \Omega) \cap H(\operatorname{div} ; \Omega), \\
(q, \nabla \cdot \boldsymbol{u})=0, & \forall q \in L^{2}(\Omega) .
\end{array}
$$

In any case, the solution to the problem is $p=0$ provided $\nabla \cdot \boldsymbol{f}=0$. This curl-div formulation admits an alternative statement in which the scalar $p$ is eliminated:

curl-div formulation II. Find $\boldsymbol{u} \in H_{0}($ curl; $\Omega) \cap H(\operatorname{div} ; \Omega)$ such that

$$
\lambda(\nabla \times \boldsymbol{u}, \nabla \times \boldsymbol{v})+\lambda(\nabla \cdot \boldsymbol{u}, \nabla \cdot \boldsymbol{v})=(\boldsymbol{f}, \boldsymbol{v}), \quad \forall \boldsymbol{v} \in H_{0}(\mathbf{c u r l} ; \Omega) \cap H(\operatorname{div} ; \Omega) .
$$

Even though both the curl and the curl-div formulations are well posed, the latter has an important drawback when considering the finite element approximation which will be described in the following subsection. This is why we will be mainly interested in the curl formulation. The norms to be considered in $V_{M 1}$ and $Q_{M 1}$ are

$$
\|\boldsymbol{v}\|_{V_{M 1}}:=\frac{1}{L_{0}}\|\boldsymbol{v}\|+\|\nabla \times \boldsymbol{v}\|, \quad\|q\|_{Q_{M 1}}:=\frac{1}{L_{0}}\|q\|+\|\nabla q\|
$$

where $L_{0}$ is a length scale that depends on the domain $\Omega$ and/or the data. Its choice is in principle arbitrary, but it will play a major role in the approximation of Darcy's problem.

The norm associated to the product space $V_{M 1} \times Q_{M 1}$ will be denoted by

$$
\|[\boldsymbol{v}, q]\|_{M}=\lambda^{\frac{1}{2}}\|\boldsymbol{v}\|_{V_{M 1}}+L_{0} \lambda^{-\frac{1}{2}}\|q\|_{Q_{M 1}} .
$$

It is important to remark that the solution to the problem satisfies $\nabla \cdot \boldsymbol{u}=0$, but the norm $\|[\boldsymbol{u}, p]\|_{M}$ provides no control on the divergence of $\boldsymbol{u}$.

Let us finally consider Darcy's problem. In this case, both functional settings indicated in Table 1 can be approximated numerically. The resulting problems are: 


\subsubsection{Maxwell's problem}

The Galerkin finite element approximation of the Maxwell problem is much more delicate than that of the Stokes problems. Suppose that we wish to approximate spaces $V_{M}$ (either $V_{M 1}$ or $V_{M 2}$ ) and $Q_{M}$ (either $Q_{M 1}$ or $Q_{M 2}$ ) by nodal finite element interpolations, defined as

$$
\mathcal{N}_{k}(\Omega)=\left\{\boldsymbol{v}_{h} \in \mathcal{C}^{0}(\Omega) \text { such that }\left.\boldsymbol{v}_{h}\right|_{K} \in \mathcal{P}_{k}(K) \forall K \in \mathcal{T}_{h}\right\}
$$

where $\mathcal{T}_{h}$ is a triangulation of the domain $\Omega$.

The crucial point in the approximation of Maxwell's problem is the difficulty to use the curl-div formulation (12), which consists of finding $\boldsymbol{u}_{h} \in V_{M 2, h}$ such that

$$
\lambda\left(\nabla \times \boldsymbol{u}_{h}, \nabla \times \boldsymbol{v}_{h}\right)+\lambda\left(\nabla \cdot \boldsymbol{u}_{h}, \nabla \cdot \boldsymbol{v}_{h}\right)=\left(\boldsymbol{f}, \boldsymbol{v}_{h}\right), \quad \forall \boldsymbol{v}_{h} \in V_{M 2, h},
$$

where $V_{M 2, h}$ is a finite element subspace of $V_{M 2}$. Finite dimensional spaces made of nodal based (or Lagrangian) finite elements are $H^{1}$-conforming. These approximations encounter the so-called corner paradox, which relies on the following result:

Lemma 1. If $\Omega$ is not convex, $V_{M 2} \cap H^{1}(\Omega)^{d}$ is a closed proper subspace of $V_{M 2}=H_{0}(\operatorname{curl} ; \Omega) \cap H(\operatorname{div} ; \Omega)$.

We also refer to the works $[13,17,14]$ for a further discussion. Thus, the solution of the continuous problem $\boldsymbol{u}$ might be outside $H^{1}(\Omega)^{d}$ if $\Omega$ is not convex. As a consequence, we have that

Corollary 1. If $\Omega$ is not convex and the finite element solutions $\boldsymbol{u}_{h}$ are uniformly bounded in $H^{1}(\Omega)^{d}$ then

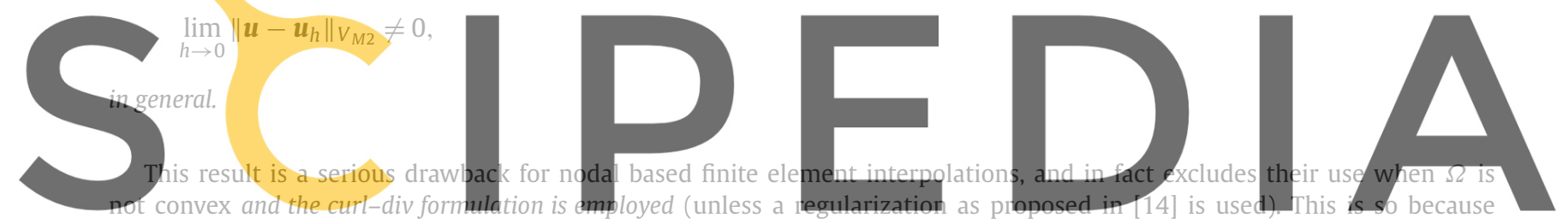

of the well known fact that

\section{Register for free at https//www.scipedia.com to download the version without the watermark \\ If $\boldsymbol{u}_{h} \in H^{1}(\Omega)^{a} \Rightarrow\left\|\nabla \boldsymbol{u}_{h}\right\| \lesssim\left\|\nabla \times \boldsymbol{u}_{h}\right\|+\left\|\nabla \cdot \boldsymbol{u}_{h}\right\|$}

Here and in the following the symbol $\lesssim$ is used to denote upper bounds up to constants ( $\gtrsim$ will be used to denote lower bounds up to constants). Since the curl-div formulation will allow us to bound the right-hand side (RHS) of this inequality (as it is trivially checked) and nodal approximations are $H^{1}(\Omega)^{d}$-conforming, the finite element solution will be uniformly bounded in $H^{1}(\Omega)^{d}$ and therefore it will be unable to approximate the so-called singular solutions (those belonging to $V_{M 2}$ but not to $\left.H^{1}(\Omega)^{d}\right)$. In general, what can be shown is that (see e.g. [13]):

$$
L_{0}^{r-1}\|\boldsymbol{u}\|_{H^{r}(\Omega)} \lesssim\|\nabla \times \boldsymbol{u}\|+\|\nabla \cdot \boldsymbol{u}\|, \quad r>1 / 2
$$

From this discussion it is sometimes said that $H^{1}(\Omega)^{d}$ conforming finite element spaces cannot approximate $V_{M 2}$ if $\Omega$ is not convex unless a weighted regularization is used (cf. [7], see also references therein for alternatives). This is a wrong conclusion, since the correct one, in view of the results stated, is that if $\left\|\nabla \cdot \boldsymbol{u}_{h}\right\|$ is uniformly bounded, $H^{1}(\Omega)^{d}$-conforming finite element spaces cannot approximate $V_{M 2}$, but only $V_{M 2} \cap H^{1}(\Omega)^{d}$. Thus, nodal interpolations are excluded only when the curl-div form of the problem (12) is being approximated (see [5]).

Let us consider now the curl formulation given by (8)-(9). The discrete version consists in finding $\boldsymbol{u}_{h} \in V_{M 1, h}$ and $p_{h} \in Q_{M 1, h}$ such that

$$
\begin{array}{ll}
a_{M}\left(\boldsymbol{u}_{h}, \boldsymbol{v}_{h}\right)+\left(\nabla p_{h}, \boldsymbol{v}_{h}\right)=\left(\boldsymbol{f}, \boldsymbol{v}_{h}\right), & \forall \boldsymbol{v}_{h} \in V_{M 1, h}, \\
-\left(\nabla q_{h}, \boldsymbol{u}_{h}\right)=0, & \forall q_{h} \in Q_{M 1, h} .
\end{array}
$$

The discrete counterpart of (13) needs to hold in order to have a well posed problem. As for the Stokes problem, convenient equal-order interpolations using nodal approximations are excluded. A possible way to design a compatible pair $V_{M 1, h} \times$ $Q_{M 1, h}$ is to construct $V_{M 1, h}$ using Nédélec's elements and $Q_{M 1, h}$ using a Lagrangian nodal interpolation (and therefore continuous). 
for all $\left[\boldsymbol{v}_{h}, q_{h}\right] \in V_{S, h} \times Q_{S, h}$, where the bilinear form $B_{S, h}$ and the linear form $L_{S, h}$ depend on the stabilized method to be considered as described in the following.

The first two methods that we consider are consistent. This is attained by introducing stabilization terms based on the magnitude of the residual.

Algebraic subgrid scale (ASGS) method. The forms $B_{S, h}$ and $L_{S, h}$ are given by:

$$
\begin{aligned}
B_{S, h}\left(\left[\boldsymbol{u}_{h}, p_{h}\right],\left[\boldsymbol{v}_{h}, q_{h}\right]\right)= & B_{S}\left(\left[\boldsymbol{u}_{h}, p_{h}\right],\left[\boldsymbol{v}_{h}, q_{h}\right]\right)+\tau_{p} \sum_{K}\left\langle\nabla \cdot \boldsymbol{u}_{h}, \nabla \cdot \boldsymbol{v}_{h}\right\rangle_{K} \\
& +\tau_{u} \sum_{K}\left\langle-v \Delta \boldsymbol{u}_{h}+\nabla p_{h}, v \Delta \boldsymbol{v}_{h}+\nabla q_{h}\right\rangle_{K}, \\
L_{S, h}\left(\left[\boldsymbol{v}_{h}, q_{h}\right]\right)=\left(\boldsymbol{f}, \boldsymbol{v}_{h}\right)+ & \tau_{u} \sum_{K}\left\langle\boldsymbol{f}, v \Delta \boldsymbol{v}_{h}+\nabla q_{h}\right\rangle_{K},
\end{aligned}
$$

where $\tau_{p}$ and $\tau_{u}$ are the so-called stabilization parameters, that we compute as

$$
\tau_{p}=c_{1} \nu, \quad \tau_{u}=\left(c_{1} \nu\right)^{-1} h^{2},
$$

with $c_{1}$ an algorithmic constant. The stabilization parameters are the same for the three methods presented.

Skew symmetric weighting (SSW) method. The forms $B_{S, h}$ and $L_{S, h}$ are given by:

$$
B_{S, h}\left(\left[\boldsymbol{u}_{h}, p_{h}\right],\left[\boldsymbol{v}_{h}, q_{h}\right]\right)=B_{S}\left(\left[\boldsymbol{u}_{h}, p_{h}\right],\left[\boldsymbol{v}_{h}, q_{h}\right]\right)+\tau_{p} \sum_{K}\left\langle\nabla \cdot \boldsymbol{u}_{h}, \nabla \cdot \boldsymbol{v}_{h}\right\rangle_{K}
$$

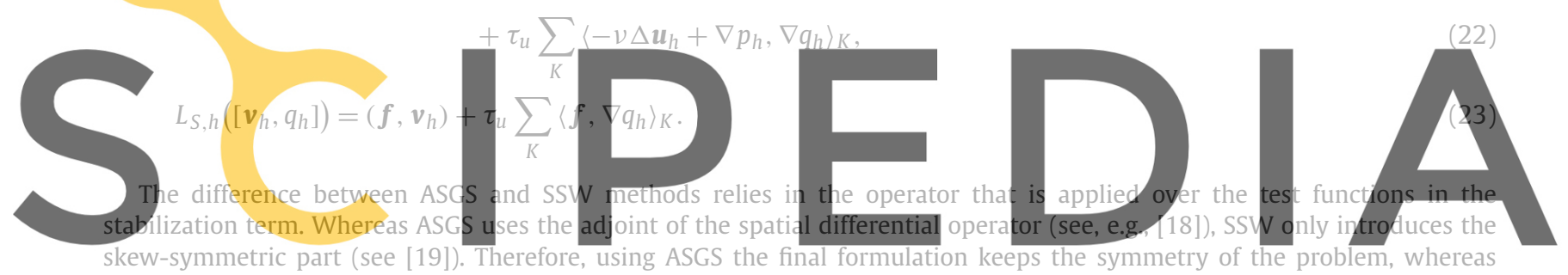

Skew-symmetric part (see [19]). Therefore, using ASGS the final formulation keeps the symmetry of the problem, whereas

the SSW method looses this symmetry. The analysis of these two methods is almost identical.

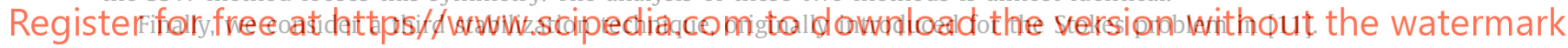

Orthogonal subscales (OSS) method. The bilinear form $B_{S, h}$ and the linear form $L_{S, h}$ in the OSS method are given by

$$
\begin{aligned}
& B_{S, h}\left(\left[\boldsymbol{u}_{h}, p_{h}\right],\left[\boldsymbol{v}_{h}, q_{h}\right]\right)=B_{S}\left(\left[\boldsymbol{u}_{h}, p_{h}\right],\left[\boldsymbol{v}_{h}, q_{h}\right]\right)+\tau_{p} \sum_{K}\left\langle P_{h}^{\perp}\left(\nabla \cdot \boldsymbol{u}_{h}\right), P_{h}^{\perp}\left(\nabla \cdot \boldsymbol{v}_{h}\right)\right\rangle_{K} \\
& +\tau_{u} \sum_{K}\left\langle P_{h}^{\perp}\left(-v \Delta \boldsymbol{u}_{h}+\nabla p_{h}\right), P_{h}^{\perp}\left(v \Delta \boldsymbol{v}_{h}+\nabla q_{h}\right)\right\rangle_{K}, \\
& L_{S, h}\left(\left[\boldsymbol{v}_{h}, q_{h}\right]\right)=\left(\boldsymbol{f}, \boldsymbol{v}_{h}\right),
\end{aligned}
$$

where $P^{\perp}=I-P_{h}, P_{h}$ being the projection onto the finite element space (for implementation issues of this projection, see [12]). Alternatively, we could consider an SSW-type OSS method, i.e. to consider only the skew-symmetric part of the spatial differential operator in the stabilization term. In this case, the viscous terms can be dropped without altering consistency, and the resulting method would be symmetric (see [10]).

Analysis. The ASGS, SSW and OSS methods have the same stability and convergence properties, even though their numerical behavior on a fixed mesh is different. Let us define the mesh dependent norm:

$$
\left\|\left[\boldsymbol{v}_{h}, q_{h}\right]\right\|_{S, h}^{2}=v\left\|\nabla \boldsymbol{v}_{h}\right\|^{2}+\frac{1}{v}\left\|q_{h}\right\|^{2}+\frac{h^{2}}{v} \sum_{K}\left\|\nabla q_{h}\right\|_{K}^{2} .
$$

In fact, the last term is unnecessary, since the first two are already those that appear in the norm of the continuous problem. However, stability in the first and the last term is in fact what the method provides, and the second term can be recovered a posteriori (see [19]). Because of this, and to clarify the generalization to be introduced in Section 3, we have decided to keep the whole expression (24).

We also define the error function 


$$
E_{S}^{2}(h)=v \varepsilon_{1}^{2}(\boldsymbol{u})+\frac{1}{v} \varepsilon_{0}^{2}(p)
$$

where $\varepsilon_{i}(\cdot)$ denotes the interpolation error in the $H^{i}(\Omega)$-seminorm. It can be proved that (24) and (25) are the norm and error function of the ASGS, the SSW and the OSS methods for the Stokes problem. This is what the following results state, that have been proved in [19] for SSW and in [11] for OSS:

Theorem 1 (Stability). Suppose that the constant $c_{1}$ is large enough. Then, there exists a constant $C>0$ such that

$$
\inf _{\left[\boldsymbol{u}_{h}, p_{h}\right] \in V_{S, h} \times Q_{S, h} \backslash\{\boldsymbol{0}, 0\}} \sup _{\left[\boldsymbol{v}_{h}, q_{h}\right] \in V_{S, h} \times Q_{S, h} \backslash\{\boldsymbol{0}, 0\}} \frac{B_{S, h}\left(\left[\boldsymbol{u}_{h}, p_{h}\right],\left[\boldsymbol{v}_{h}, q_{h}\right]\right)}{\left\|\left[\boldsymbol{u}_{h}, p_{h}\right]\right\|_{S, h}\left\|\left[\boldsymbol{v}_{h}, q_{h}\right]\right\|_{S, h}} \geqslant C>0 .
$$

Theorem 2 (Convergence). Let $[\boldsymbol{u}, p]$ be the solution of the continuous problem and $\left[\boldsymbol{u}_{h}, p_{h}\right]$ the solution of the discrete one. Suppose as before that $c_{1}$ is large enough. Then

$$
\left\|\left[\boldsymbol{u}-\boldsymbol{u}_{h}, p-p_{h}\right]\right\|_{S, h} \lesssim E_{S}(h) .
$$

\subsubsection{Maxwell's problem}

The stabilized finite element method we propose has recently been introduced in [5], with slight modifications. In the original article, the pressure stabilization makes use of the fact that $p=0$ for a solenoidal $\boldsymbol{f}$, by stating the problem in a novel augmented formulation that provides pressure stability. In the following we will motivate the method as a residual-based stabilized finite element method, and we will also design a new OSS technique for the problem at hand. This reinterpretation allows us to couple the resulting residual-based stabilized method with those developed for Stokes' and Darcy's problems.

In the following, we assume that $V_{M} \equiv V_{M 1}$ and $Q_{M} \equiv Q_{M 1}$, defined in Table 1 ; we only consider the curl functional setting that allows one to approximate singular solutions. Let $V_{M, h}$ and $Q_{M, h}$ be finite element approximations of $V_{M}$ and $Q_{M}$ respectively. We can now state the ASGS and OSS stabilized algorithms for Maxwell's problem as follows: find $\left[\boldsymbol{u}_{\boldsymbol{h}}, p_{h}\right] \in V_{M, h} \times Q_{M, h}$ such that $B_{M, h}\left(\left[\boldsymbol{u}_{h}, p_{h}\right],\left[\boldsymbol{v}_{h}, q_{h}\right]\right)$ $11\left[v_{h}, q_{h}\right] \in V_{M, h} \times Q_{M, h}, w$ $B_{M, h}\left(\left[\boldsymbol{u}_{h}, p_{h}\right],\left[v_{h}, q_{h}\right]\right)=B_{M}\left(\left[\boldsymbol{u}_{h}, \boldsymbol{p}_{h}\right],\left[v_{h}, q_{h}\right]\right)+\tau_{h}\left(\tilde{P}\left(\nabla \cdot \boldsymbol{u}_{h}\right), \nabla \cdot v_{h}\right.$
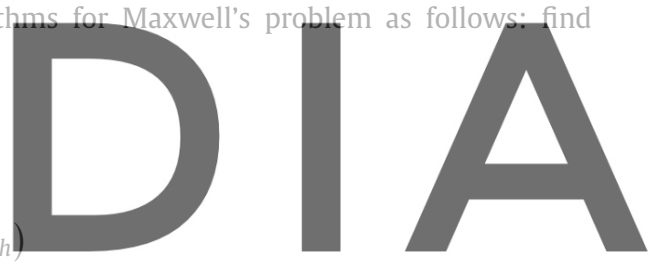

$$
+\sum \tau_{u}\left\langle\tilde{P}\left(\lambda \nabla \times \nabla \times \boldsymbol{u}_{h}+\nabla p_{h}\right),-\lambda \nabla \times \nabla \times v_{h}+\nabla q_{h}\right\rangle_{K}
$$

(26) Register for free at https//www.\$cipedia.com to download the version without the watermark

$$
L_{M, h}\left(\left[v_{h}, q_{h}\right]\right)=\left(\boldsymbol{f}, v_{h}\right)+\sum_{K} \tau_{u}\left(\tilde{P}(f),-\lambda \nabla \times \nabla \times v_{h}+\nabla q_{h}\right\rangle_{K},
$$

and the stabilization parameters read:

$$
\tau_{p}=c_{2} \lambda \frac{h^{2}}{L_{0}^{2}}, \quad \tau_{u}=\frac{L_{0}^{2}}{\lambda}
$$

Apart from the definition of $L_{0}, c_{2}$ is the algorithmic constant on which the formulation depends. The projection $\tilde{P}$ can be either the identity $I$ or the projection orthogonal to the finite element space $P \stackrel{\perp}{\perp}$, getting the ASGS and OSS formulations, respectively.

When taking the test functions equal to the unknowns to prove stability we get

$$
B_{M, h}\left(\left[\boldsymbol{u}_{h}, p_{h}\right],\left[\boldsymbol{u}_{h}, p_{h}\right]\right)=\lambda\left\|\nabla \times \boldsymbol{u}_{h}\right\|^{2}-L_{0}^{2} \lambda\left\|\tilde{P}\left(\nabla \times \nabla \times \boldsymbol{u}_{h}\right)\right\|^{2}+\frac{L_{0}^{2}}{\lambda}\left\|\tilde{P}\left(\nabla p_{h}\right)\right\|^{2}+c_{2} \lambda \frac{h^{2}}{L_{0}^{2}}\left\|\tilde{P}\left(\nabla \cdot \boldsymbol{u}_{h}\right)\right\|^{2} .
$$

The problem is the control of the second term. When $\tilde{P}=I$, this control could be achieved using an inverse inequality, but for that $L_{0}$ should behave as $h$, and this would not allow us to approximate singular solutions (see [5]). For these reasons, the previous algorithm is discarded.

Skew symmetric weighting method. Instead we can consider an SSW-type formulation, in which the bilinear form of the problem is given by

$$
\begin{aligned}
B_{M, h}\left(\left[\boldsymbol{u}_{h}, p_{h}\right],\left[\boldsymbol{v}_{h}, q_{h}\right]\right)= & B_{M}\left(\left[\boldsymbol{u}_{h}, p_{h}\right],\left[\boldsymbol{v}_{h}, q_{h}\right]\right) \\
& +\sum_{K} \frac{L_{0}^{2}}{\lambda}\left\langle\lambda \nabla \times \nabla \times \boldsymbol{u}_{h}+\nabla p_{h}, \nabla q_{h}\right\rangle_{K}+\sum_{K} c_{2} \lambda \frac{h^{2}}{L_{0}^{2}}\left\langle\nabla \cdot \boldsymbol{u}_{h}, \nabla \cdot \boldsymbol{v}_{h}\right\rangle_{K} .
\end{aligned}
$$


Since $\sum_{K}\left\langle\lambda \nabla \times \nabla \times \boldsymbol{u}_{h}, \nabla q_{h}\right\rangle_{K}=0$, this term can be canceled out, recovering the same method as in [5]:

$$
\begin{aligned}
& B_{M, h}\left(\left[\boldsymbol{u}_{h}, p_{h}\right],\left[\boldsymbol{v}_{h}, q_{h}\right]\right)=B_{M}\left(\left[\boldsymbol{u}_{h}, p_{h}\right],\left[\boldsymbol{v}_{h}, q_{h}\right]\right)+\sum_{K} \frac{L_{0}^{2}}{\lambda}\left\langle\nabla p_{h}, \nabla q_{h}\right\rangle_{K}+\sum_{K} c_{2} \lambda \frac{h^{2}}{L_{0}^{2}}\left\langle\nabla \cdot \boldsymbol{u}_{h}, \nabla \cdot \boldsymbol{v}_{h}\right\rangle_{K}, \\
& L_{M, h}\left(\left[\boldsymbol{v}_{h}, q_{h}\right]\right)=\left(\boldsymbol{f}, \boldsymbol{v}_{h}\right)+\sum_{K} \frac{L_{0}^{2}}{\lambda}\left\langle\boldsymbol{f}, \nabla q_{h}\right\rangle_{K} .
\end{aligned}
$$

We stress the fact that this SSW stabilization ends up being symmetric. Note that the last term vanishes if $\boldsymbol{f}$ is solenoidal.

Orthogonal subscales method. When $\tilde{P}=P \frac{\perp}{h}$, the term $P_{h}^{\perp}\left(\lambda \nabla \times \nabla \times \boldsymbol{u}_{h}\right)$ in (26)-(27) can be omitted without sacrificing accuracy. If this is done, the bilinear form of the problem is

$$
\begin{aligned}
B_{M, h}\left(\left[\boldsymbol{u}_{h}, p_{h}\right],\left[\boldsymbol{v}_{h}, q_{h}\right]\right)= & B_{M}\left(\left[\boldsymbol{u}_{h}, p_{h}\right],\left[\boldsymbol{v}_{h}, q_{h}\right]\right) \\
& +\sum_{K} \frac{L_{0}^{2}}{\lambda}\left\langle P_{h}^{\perp}\left(\nabla p_{h}\right), P_{h}^{\perp}\left(\nabla q_{h}\right)\right\rangle_{K}+\sum_{K} c_{2} \lambda \frac{h^{2}}{L_{0}^{2}}\left\langle P_{h}^{\perp}\left(\nabla \cdot \boldsymbol{u}_{h}\right), P_{h}^{\perp}\left(\nabla \cdot \boldsymbol{v}_{h}\right)\right\rangle_{K} .
\end{aligned}
$$

Analysis. We will not analyze in detail the formulations determined by (29) and (30), but the following discussion will be useful in Section 3 to design the combined problem. Let us state the stability and convergence results we have proved for problem (29) in [5]. First, let us define the mesh-dependent norm:

$$
\left\|\left[\boldsymbol{v}_{h}, q_{h}\right]\right\|_{M, h}=\lambda^{\frac{1}{2}}\left\|\nabla \times \boldsymbol{v}_{h}\right\|+\lambda^{\frac{1}{2}} \frac{h}{L_{0}}\left\|\nabla \cdot \boldsymbol{u}_{h}\right\|+\frac{L_{0}}{\lambda^{\frac{1}{2}}}\left\|\nabla p_{h}\right\| .
$$

As for the Stokes problem, this is the norm for which the method provides stability in a straightforward manner. Indeed, it is ezsy to check that the SSW niethod is coercive with respect to this norm. For OSS, we immediately get we then have

Register for free at https//www.scipedia.com to dowaload the version without the watermark

$B_{M, h}\left(\left[\boldsymbol{u}_{h}, p_{h}\right],\left[\boldsymbol{v}_{h, 0}, 0\right]\right) \geq-\ell^{2}\left\|\nabla \times \boldsymbol{u}_{h}\right\|\left\|\nabla \times P_{h}\left(\nabla p_{h}\right)\right\|+\frac{\ell}{\lambda}\left\|P_{h}\left(\nabla p_{h}\right)\right\|^{2}-\frac{\ell^{2}}{L_{0}^{2}}\left\|\nabla \cdot \boldsymbol{u}_{h}\right\|\left\|\nabla \cdot P_{h}\left(\nabla p_{h}\right)\right\|$

$$
\begin{aligned}
\gtrsim & -\frac{\beta_{1}}{2} \lambda\left\|\nabla \times \boldsymbol{u}_{h}\right\|^{2}-\frac{1}{2 \beta_{1}} \frac{\ell^{4}}{\lambda h^{2}}\left\|P_{h}\left(\nabla p_{h}\right)\right\|^{2}+\frac{\ell^{2}}{\lambda}\left\|P_{h}\left(\nabla p_{h}\right)\right\|^{2} \\
& -\frac{\beta_{2}}{2} \frac{\lambda h^{2}}{L_{0}^{2}}\left\|\nabla \cdot \boldsymbol{u}_{h}\right\|^{2}-\frac{1}{2 \beta_{2}} \frac{\ell^{4}}{\lambda L_{0}^{2}}\left\|P_{h}\left(\nabla p_{h}\right)\right\|^{2},
\end{aligned}
$$

which holds for arbitrary $\beta_{1}, \beta_{2}>0$. The optimal situation would be to take $\ell=L_{0}$, but then we cannot control the second term of the last bound. To be able to achieve this control, we must take $\ell=h$. On the other hand, full stability over $\nabla \cdot \boldsymbol{u}_{h}$ is attained taking as test function $q_{h, 0}=\frac{\lambda h^{2}}{L_{0}^{2}} P_{h}\left(\nabla \cdot \boldsymbol{u}_{h}\right)$, getting:

$$
\begin{aligned}
B_{M, h}\left(\left[\boldsymbol{u}_{h}, p_{h}\right],\left[\mathbf{0}, q_{h, 0}\right]\right) & \gtrsim \frac{\lambda h^{2}}{L_{0}^{2}}\left\|P_{h}\left(\nabla \cdot \boldsymbol{u}_{h}\right)\right\|^{2}-\left\|\nabla p_{h}\right\| h^{2}\left\|\nabla P_{h}\left(\nabla \cdot \boldsymbol{u}_{h}\right)\right\| \\
& \gtrsim \frac{\lambda h^{2}}{L_{0}^{2}}\left\|P_{h}\left(\nabla \cdot \boldsymbol{u}_{h}\right)\right\|^{2}-\frac{L_{0}^{2}}{2 \beta_{3} \lambda}\left\|\nabla p_{h}\right\|^{2}-\frac{\lambda h^{2}}{2 \beta_{3} L_{0}^{2}}\left\|P_{h}\left(\nabla \cdot \boldsymbol{u}_{h}\right)\right\|^{2}
\end{aligned}
$$

for an arbitrary $\beta_{3}>0$. After combining these results, it is not difficult to see that the formulation obtained using the bilinear form (30) is stable (in the form of an inf-sup condition) in the norm

$$
\left\|\left[\boldsymbol{v}_{h}, q_{h}\right]\right\|_{M, h}=\lambda^{\frac{1}{2}}\left\|\nabla \times \boldsymbol{v}_{h}\right\|+\lambda^{\frac{1}{2}} \frac{h}{L_{0}}\left\|\nabla \cdot \boldsymbol{u}_{h}\right\|+\frac{L_{0}}{\lambda^{\frac{1}{2}}}\left\|P_{h}^{\perp}\left(\nabla p_{h}\right)\right\|+\frac{h}{\lambda^{\frac{1}{2}}}\left\|P_{h}\left(\nabla p_{h}\right)\right\|,
$$

which is weaker than (31). It is worthy to note that control on the last term can also be obtained for a Galerkin formulation.

In the following, we list some results proved in [5]: 
Lemma 2 (Stability in the mesh dependent norm). The bilinear form in (29), $B_{M, h}: V_{M, h} \times Q_{M, h} \times V_{M, h} \times Q_{M, h} \rightarrow \mathbb{R}$, is coercive with respect to the mesh-dependent norm (31).

However, once stability is proved in this norm, it can also be proved in the norm defined in Table 2 for the Maxwell problem:

Lemma 3 (Norm equivalence). The solution $\left[\boldsymbol{w}_{h}, \alpha_{h}\right] \in V_{M, h} \times Q_{M, h}$ of the discrete problem

$$
B_{M, h}\left(\boldsymbol{w}_{h}, \alpha_{h} ; \boldsymbol{v}_{h}, q_{h}\right)=\left\langle\boldsymbol{f}, \boldsymbol{v}_{h}\right\rangle+\left\langle g, q_{h}\right\rangle, \quad \forall\left(\boldsymbol{v}_{h}, q_{h}\right) \in V_{M, h} \times Q_{M, h},
$$

for $\boldsymbol{f} \in V^{\prime}$ and $g \in Q^{\prime}$, satisfies:

$$
\left\|\boldsymbol{w}_{h}, \alpha_{h}\right\|_{M, h} \lesssim\left\|\boldsymbol{w}_{h}, \alpha_{h}\right\|_{M} \lesssim\left\|\boldsymbol{w}_{h}, \alpha_{h}\right\|_{M, h}+\|g\|_{Q^{\prime}} .
$$

The idea to prove this result is to recover the $L^{2}(\Omega)$-control on $\boldsymbol{u}$ and $p$ from the control on the divergence of $\boldsymbol{u}$ and the gradient of $p$, respectively. To this end, the continuous inf-sup condition is crucial. Note that in this case we have considered an arbitrary function $g$. This allows us to obtain the target stability result:

Corollary 2 (Natural stability). The solution $\left[\boldsymbol{u}_{h}, p_{h}\right]$ of the problem satisfies

$$
\left\|\left[\boldsymbol{u}_{h}, p_{h}\right]\right\|_{M} \lesssim\|\boldsymbol{f}\| .
$$

When restricted to the discrete finite element spaces, the bilinear form of our method is continuous:

Corollary 3 (Natural continuity). The stabilized bilinear form in (29), $B_{M, h}: V_{M, h} \times Q_{M, h} \times V_{M, h} \times Q_{M, h} \rightarrow \mathbb{R}$, is continuous with respect to the norm $\|\cdot\|_{M}$. by

These results allow us to prove a convergence result in a straightforward way. The error function of the method is given

$$
E_{M}(h):=\inf _{\left[\boldsymbol{w}_{h}, r_{h}\right] \in V_{M, h} \times Q_{M, h}}\left[\left\|\left[\boldsymbol{u}-\boldsymbol{w}_{h}, p-r_{h}\right]\right\|_{M}+\lambda^{\frac{1}{2}}\left(\sum_{K} \frac{h}{L_{0}^{2}}\left\|\boldsymbol{u}-\boldsymbol{w}_{h}\right\|_{L^{2}(\partial K)}^{2}\right)^{\frac{1}{2}}\right] .
$$

We have left the last term, which shows the additional error introduced by our method compared to what could be expected from a Galerkin approach. Nevertheless, this last term behaves in an optimal way. We have:

Theorem 3 (Convergence). The solution $\left[\boldsymbol{u}_{h}, p_{h}\right]$ of the discrete problem satisfies

$$
\left\|\left[\boldsymbol{u}_{h}-\boldsymbol{u}, p_{h}-p\right]\right\|_{M} \lesssim E_{M}(h) .
$$

In Maxwell's problem it is important to clearly state how the method converges when the solutions are smooth and when they are not, since singular solutions are common in non-convex domains. In general, we have the following interpolation estimates:

$$
\begin{array}{cl}
\inf _{\boldsymbol{w}_{h} \in V_{M, h}}\left\|\boldsymbol{v}-\boldsymbol{w}_{h}\right\|_{H^{s}(\omega)} \lesssim h^{t-s}\|\boldsymbol{v}\|_{H^{t}(\omega)}, & 0 \leqslant s \leqslant t \leqslant k+1, \\
\inf _{r_{h} \in Q_{M, h}}\left\|q-r_{h}\right\|_{H^{s}(\omega)} \lesssim h^{t-s}\|q\|_{H^{t}(\omega)}, & 0 \leqslant s \leqslant t \leqslant l+1,
\end{array}
$$

for any bounded set $\omega \subset \Omega$.

When the solution is smooth we have, from the last convergence theorem:

Corollary 4 (Convergence to smooth solutions). If $\boldsymbol{u} \in H^{r}(\Omega)^{d}$, with $r \geqslant 1$, the solution $\left[\boldsymbol{u}_{h}, p_{h}\right]$ satisfies:

$$
\left\|\boldsymbol{u}-\boldsymbol{u}_{h}, p-p_{h}\right\|_{M} \lesssim \lambda^{\frac{1}{2}} h^{t-1}\|\boldsymbol{u}\|_{H^{t}(\Omega)}, \quad t:=\min \{r, k+1\} .
$$

This is clearly an optimal result. For singular solutions we have to make use of the following result:

Lemma 4 (Decomposition of singular solutions). The solution $\boldsymbol{u} \in V_{M} \cap H(\mathrm{div} ; \Omega$ ) of the problem can be decomposed into a regular part and a singular part as follows:

$$
\boldsymbol{u}=\boldsymbol{u}_{0}+\nabla \varphi,
$$

where $\boldsymbol{u}_{0} \in H^{1+r}(\Omega)^{d} \cap H_{0}(\mathbf{c u r l} ; \Omega), \varphi \in H_{0}^{1}(\Omega) \cap H^{1+r}(\Omega)$ for some real number $r>\frac{1}{2}$. 

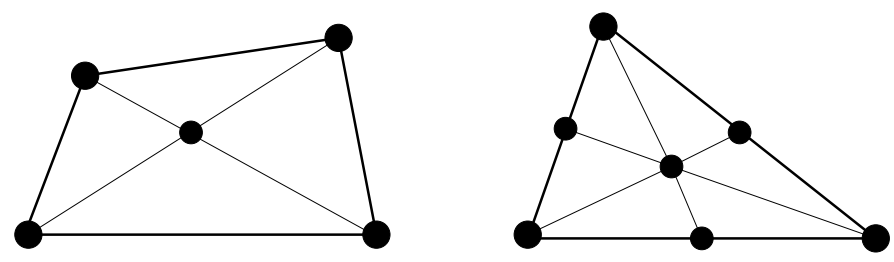

Fig. 2. Crossed-box (left) and Powell-Sabin (right) macro-element typologies.

In order to prove convergence to singular solutions, we have to assume that the finite element partition is able to approximate the gradient part of this solution. This assumption is common to other numerical formulations and can be stated as follows:

Assumption 1. There exists a finite element space $G_{h}$ defined over $\mathcal{T}_{h}$ such that, for any $\phi_{h} \in G_{h}, \nabla \phi_{h} \in V_{M, h}$. Furthermore, this space satisfies

$$
\inf _{\phi_{h} \in G_{h}}\left\|\phi-\phi_{h}\right\|_{H^{s}(\omega)} \lesssim h^{t-s}\|\phi\|_{H^{t}(\omega)}
$$

for $\phi \in H^{t}(\omega)$ and $0 \leqslant s \leqslant t \leqslant 1+k$.

Assumption 1 is known to hold for interpolation orders $k \geqslant 4$ in dimension 2 without any assumption on the mesh typology. In this case, we can take $G_{h}$ as the finite element space obtained for the Argyris triangle. For $k \geqslant 2, G_{h}$ can be constructed by using the Bogner-Fox-Schmidt triangle; in order to do this, the triangulation $\mathcal{T}_{h}$ should admit a coarser mesh of macro-elements. We refer to [14] for a detailed discussion.

The discrete space recently introduced in [25], based on a Powell-Sabin interpolant (see Fig. 2 right), makes true Assumption 1 for $k \geqslant 1$, both in two and three dimensions. Furthermore, we have observed from numerical experiments that a mesh with the crossed-box typology (see Fig. 2 left) also satisfies this assumption. In a numerical code, it implies to perform a cheap pre-processing of the original mesh. Given any original triangular mesh, the Powell-Sabin mesh is obtained by introducing additional nodes on the mid-points of the edges and the element barycenters, and re-connecting the nodes properly. On the other hand, crossed-box meshes are obtained from a quadrilateral mesh by placing a node on its center, and creating four triangles; in fact, the additional node can be condensed.

If Assumption 1 holds, we are able to prove the following quasi-optimal error estimate [5]:

Corollary 5 (Convergence to singular solutions). Under Assumption 1, the solution $\left[\boldsymbol{u}_{h}, p_{h}\right]$ of the discrete problem satisfies

$$
\left\|\boldsymbol{u}-\boldsymbol{u}_{h}, p-p_{h}\right\|_{M} \lesssim \lambda^{\frac{1}{2}} h^{t}\left\|\boldsymbol{u}_{0}\right\|_{H^{1+t}(\Omega)}+\frac{\lambda^{\frac{1}{2}}}{L_{0}^{1-\epsilon}} h^{t-\epsilon}\|\varphi\|_{H^{1+t}(\Omega)},
$$

for any $\epsilon \in] 0, t-1 / 2[$ and for $t=\min \{r, k\}$, where $r$ is defined in Lemma 4 .

This result is based on the embedding of $H^{\epsilon}(\partial K)$ in $H^{\epsilon+\frac{1}{2}}(K)$ for any element domain $K$, and therefore the bound explodes as $\epsilon \rightarrow 0$.

\subsubsection{Darcy's problem}

The formulation of the method we propose and its analysis for Darcy's problem follows exactly the same lines as for the Stokes problem. Let $V_{D, h}$ and $Q_{D, h}$ be the finite element spaces to approximate the velocity and the pressure, respectively. The methods to be analyzed can be written as follows: find $\left[\boldsymbol{u}_{h}, p_{h}\right] \in V_{D, h} \times Q_{D, h}$ such that

$$
B_{D, h}\left(\left[\boldsymbol{u}_{h}, p_{h}\right],\left[\boldsymbol{v}_{h}, q_{h}\right]\right)=L_{D, h}\left(\left[\boldsymbol{v}_{h}, q_{h}\right]\right)
$$

for all $\left[\boldsymbol{v}_{h}, q_{h}\right] \in V_{D, h} \times Q_{D, h}$. Once again, the bilinear and linear forms of the problem are defined depending on the stabilization method being used.

Algebraic subgrid scale method. The forms $B_{D, h}$ and $L_{D, h}$ are given by:

$$
\begin{aligned}
& B_{D, h}\left(\left[\boldsymbol{u}_{h}, p_{h}\right],\left[\boldsymbol{v}_{h}, q_{h}\right]\right)= B_{D}\left(\left[\boldsymbol{u}_{h}, p_{h}\right],\left[\boldsymbol{v}_{h}, q_{h}\right]\right)+\tau_{p} \sum_{K}\left\langle\nabla \cdot \boldsymbol{u}_{h}, \nabla \cdot \boldsymbol{v}_{h}\right\rangle_{K} \\
&+\tau_{u} \sum_{K}\left\langle\sigma \boldsymbol{u}_{h}+\nabla p_{h},-\sigma \boldsymbol{v}_{h}+\nabla q_{h}\right\rangle_{K}, \\
& L_{D, h}\left(\left[\boldsymbol{v}_{h}, q_{h}\right]\right)=\left(\boldsymbol{f}, \boldsymbol{v}_{h}\right)+\left(g, q_{h}\right)+\tau_{p} \sum_{K}\left\langle g, \nabla \cdot \boldsymbol{v}_{h}\right\rangle_{K}+\tau_{u} \sum_{K}\left\langle\boldsymbol{f},-\sigma \boldsymbol{v}_{h}+\nabla q_{h}\right\rangle_{K} .
\end{aligned}
$$


The stabilization parameters are computed as

$$
\tau_{p}=c_{3} \sigma \ell^{2}, \quad \tau_{u}=\left(c_{3} \sigma \ell^{2}\right)^{-1} h^{2},
$$

with $c_{3}$ an algorithmic constant and $\ell$ a length scale to be determined. This length scale turns out to be crucial to be able to approximate either the primal or the dual form of the problem. It can be taken as $L_{0}, h$ or $\left(L_{0} h\right)^{1 / 2}$. Its introduction can be motivated by scaling arguments. In fact, the length scale in $\tau_{p}$ could be different from that in $\tau_{u}$, but we have taken them equal to simplify the discussion. Using an approximate Fourier analysis as in [4], the stabilization parameters are found, now depending on scaling coefficients $\mu_{u}$ and $\mu_{p}$. In turn, these scaling coefficients depend on a length scale of the problem that may be taken as $L_{0}$ or $h$. This is what determines the choice for $\ell$. The same expression of the stabilization parameters is used for SSW and OSS algorithms presented next.

Skew symmetric weighting method. The forms $B_{D, h}$ and $L_{D, h}$ are given by:

$$
\begin{aligned}
& B_{D, h}\left(\left[\boldsymbol{u}_{h}, p_{h}\right],\left[\boldsymbol{v}_{h}, q_{h}\right]\right)=B\left(\left[\boldsymbol{u}_{h}, p_{h}\right],\left[\boldsymbol{v}_{h}, q_{h}\right]\right)+\tau_{p} \sum_{K}\left\langle\nabla \cdot \boldsymbol{u}_{h}, \nabla \cdot \boldsymbol{v}_{h}\right\rangle_{K}+\tau_{u} \sum_{K}\left\langle\sigma \boldsymbol{u}_{h}+\nabla p_{h}, \nabla q_{h}\right\rangle_{K}, \\
& L_{D, h}\left(\left[\boldsymbol{v}_{h}, q_{h}\right]\right)=\left(\boldsymbol{f}, \boldsymbol{v}_{h}\right)+\left(g, q_{h}\right)+\tau_{p} \sum_{K}\left\langle g, \nabla \cdot \boldsymbol{v}_{h}\right\rangle_{K}+\tau_{u} \sum_{K}\left\langle\boldsymbol{f}, \nabla q_{h}\right\rangle_{K} .
\end{aligned}
$$

This formulation is non-symmetric but introduces all the stabilization needed for the discrete problem to be well-posed.

Orthogonal subscales method. The bilinear form $B_{D, h}$ and the linear form $L_{D, h}$ in the OSS method are given by

$$
\begin{aligned}
B_{D, h}\left(\left[\boldsymbol{u}_{h}, p_{h}\right],\left[\boldsymbol{v}_{h}, q_{h}\right]\right)= & B_{D}\left(\left[\boldsymbol{u}_{h}, p_{h}\right],\left[\boldsymbol{v}_{h}, q_{h}\right]\right) \\
& +\tau_{p} \sum_{K}\left\langle P_{h}^{\perp}\left(\nabla \cdot \boldsymbol{u}_{h}\right), P_{h}^{\perp}\left(\nabla \cdot \boldsymbol{v}_{h}\right)\right\rangle_{K}+\tau_{u} \sum_{K}\left\langle P_{h}^{\perp}\left(\nabla p_{h}\right), P_{h}^{\perp}\left(\nabla q_{h}\right)\right\rangle_{K}, \\
L_{D, h}\left(\left[\boldsymbol{v}_{h}, q_{h}\right]\right)=\left(\boldsymbol{f}, \boldsymbol{v}_{h}\right)+ & \left(g, q_{h}\right) .
\end{aligned}
$$

Analysis. As for the Stokes problem, all the methods have the same stability and convergence properties. Let us define the mesh dependent norm

$$
\left\|\left[\boldsymbol{v}_{h}, q_{h}\right]\right\|_{D, h}^{2}=\sigma\left\|\boldsymbol{v}_{h}\right\|^{2}+\sigma \ell^{2}\left\|\nabla \cdot \boldsymbol{v}_{h}\right\|^{2}+\frac{1}{\sigma L_{0}^{2}}\left\|q_{h}\right\|^{2}+\frac{h^{2}}{\sigma \ell^{2}} \sum_{K}\left\|\nabla q_{h}\right\|_{K}^{2},
$$

as well as the error function

$$
E_{D}^{2}(h)=\sigma \ell^{2}\left(h^{-2} \varepsilon_{0}^{2}(\boldsymbol{u})+\varepsilon_{1}^{2}(\boldsymbol{u})\right)+\sigma \varepsilon_{0}^{2}(\boldsymbol{u})+\frac{h^{2}}{\sigma \ell^{2}}\left(h^{-2} \varepsilon_{0}^{2}(p)+\varepsilon_{1}^{2}(p)\right) .
$$

The following results can be summarized by saying that these are the norm and error function of the methods introduced:

Theorem 4 (Stability). Suppose that the constant $c_{3}$ in (33) is large enough. Then, there exists a constant $C$ such that

$$
\inf _{\left[\boldsymbol{u}_{h}, p_{h}\right] \in V_{D, h} \times Q_{D, h} \backslash\{\mathbf{0}, 0\}} \sup _{\left[\boldsymbol{v}_{h}, q_{h}\right] \in V_{D, h} \times Q_{D, h} \backslash\{\boldsymbol{0}, 0\}} \frac{B_{D, h}\left(\left[\boldsymbol{u}_{h}, p_{h}\right],\left[\boldsymbol{v}_{h}, q_{h}\right]\right)}{\left\|\left[\boldsymbol{u}_{h}, p_{h}\right]\right\|\left\|_{D, h}\right\|\left[\boldsymbol{v}_{h}, q_{h}\right]\|\|_{D, h}} \geqslant C>0 .
$$

Theorem $\mathbf{5}$ (Convergence). Let $[\boldsymbol{u}, p]$ be the solution of the continuous problem and $\left[\boldsymbol{u}_{h}, p_{h}\right]$ the solution of the discrete one. Suppose as before that $c_{3}$ is large enough. Then

$$
\left\|\left[\boldsymbol{u}-\boldsymbol{u}_{h}, p-p_{h}\right]\right\|_{D, h} \lesssim E_{D}(h)
$$

We refer to [3,4] for the proof of these results. Let us discuss the implications of the choice of $\ell$ in view of the working norm in (34) and the error function in (35). On the one hand, if $\ell=h$ (up to constants), we do not have control on the velocity divergence, but pressure gradients are controlled. Thus, (34) coincides with the norm of the primal formulation of Darcy's problem in Table 2, and the error function (35) (with $\ell=h$ ) is consistent with this fact. On the other hand, if $\ell=L_{0}$ (34) is the discrete counterpart of the norm of the dual formulation of Darcy's problem in Table 2 and, as in the previous case, the error function (35) (with $\ell=L_{0}$ ) is consistent with this fact. 


\section{Combined problem}

\subsection{Boundary value problem}

In this section we consider the problem that combines all the model problems discussed heretofore. It consists of finding $\boldsymbol{u}$ and $p$ solution of the differential equations posed in a domain $\Omega$

$$
\begin{aligned}
& -v \Delta \boldsymbol{u}+\lambda \nabla \times \nabla \times \boldsymbol{u}+\sigma \boldsymbol{u}+\nabla p=\boldsymbol{f}, \\
& \nabla \cdot \boldsymbol{u}=g,
\end{aligned}
$$

together with appropriate boundary conditions on the normal and tangential components of $\boldsymbol{u}$ and, if $v=0$, also on $p$ on $\partial \Omega$ (possible if $\sigma>0$, needed if $\sigma=0$ ). The problem has obviously the structure (1)-(3), with

$$
L_{X}(\boldsymbol{u})=L_{S}(\boldsymbol{u})+L_{M}(\boldsymbol{u})+L_{D}(\boldsymbol{u}) .
$$

If $v>0$, the problem is in principle posed in the functional setting of the Stokes problem. However, at the numerical level it does matter whether $v$ is small or not compared to the rest of physical parameters. Thus, if a Galerkin method is used which is stable for the Stokes problems, results are likely to be very poor if $v$ is small. Likewise, in the case $v=0$ it is not clear which is the interpolation that will lead to a stable method, since it is different for Maxwell's problem $(\lambda>0$, $\sigma=0)$ and for Darcy's problem $(\lambda=0, \sigma>0)$.

Our goal is therefore to propose an approximation of the boundary value problem (36)-(37) (plus boundary conditions) able to deal with the limits

$$
\nu \rightarrow 0, \quad \sigma \rightarrow 0, \quad \text { or } \quad \lambda \rightarrow 0 .
$$

Concerning the physical interest of (36)-(37), the case $\lambda=0$ is known as the Brinkman problem, and models the flow of viscous fluids in porous media. The case $v=0$ is relevant to the analysis of eigenvalues of the Maxwell operator. We are not aware of any situation involving $v>0$ and $\lambda>0$, but nevertheless being able to deal with it highlights the robustness of a numerical formulation.

\subsection{Variational form and functional setting}

The weak form of problem (36)-(37), with the homogeneous boundary conditions used in the model problems, is straightforward. It consists of finding $[\boldsymbol{u}, p]$ such that

$$
B_{\mathrm{SMD}}([\boldsymbol{u}, p],[\boldsymbol{v}, q])=(\boldsymbol{f}, \boldsymbol{v})+(g, q),
$$

for all $[\boldsymbol{v}, q]$ in the appropriate functional space $V \times Q$, which is discussed next. The bilinear form $B_{\mathrm{SMD}}$ in (38) is given by

$$
B_{\mathrm{SMD}}([\boldsymbol{u}, p],[\boldsymbol{v}, q])=v(\nabla \boldsymbol{u}, \nabla \boldsymbol{v})+\lambda(\nabla \times \boldsymbol{u}, \nabla \times \boldsymbol{v})+\sigma(\boldsymbol{u}, \boldsymbol{v})+\langle\nabla p, \boldsymbol{v}\rangle+\langle q, \nabla \cdot \boldsymbol{u}\rangle .
$$

The functional setting is a delicate issue if we want to encompass all possible situations discussed previously for the model problems. We define the norm

$$
\|\boldsymbol{v}\|_{V}=L_{0}^{-1} \kappa^{\frac{1}{2}}\|\boldsymbol{v}\|+v^{\frac{1}{2}}\|\nabla \boldsymbol{v}\|+\lambda^{\frac{1}{2}}\|\nabla \times \boldsymbol{v}\|+\kappa_{\ell}^{\frac{1}{2}}\|\nabla \cdot \boldsymbol{v}\|^{2}
$$

with

$$
\kappa=v+\lambda+\sigma L_{0}^{2}, \quad \kappa_{\ell}=v \frac{\ell_{S}^{2}}{L_{0}^{2}}+\lambda \frac{\ell_{M}^{2}}{L_{0}^{2}}+\sigma \ell_{D}^{2},
$$

and where $\ell_{S}, \ell_{M}$ and $\ell_{D}$ are characteristic length scales for the Stokes, the Maxwell and the Darcy problems, respectively, which may be either $L_{0}$ or 0 . In fact, for the Stokes problem only the case $\ell_{S}=L_{0}$ makes sense, as we will see immediately. We also denote by $\|\cdot\|_{V^{\prime}}$ the corresponding dual norm.

This, in turn, allows us to define the following norm:

$$
\|q\|_{Q}:=\sup _{\boldsymbol{v} \neq \mathbf{0}} \frac{\langle\nabla q, \boldsymbol{v}\rangle}{\|\boldsymbol{v}\|_{V}} .
$$

This is the crucial ingredient we need to define the following norm:

$$
\|[\boldsymbol{v}, q]\|_{X}:=\|\boldsymbol{v}\|_{V}+\|q\|_{Q} .
$$

Finally, we define $V \times Q$ as the closure of $\mathcal{C}_{0}^{\infty}(\Omega)^{d} \times \mathcal{C}_{0}^{\infty}(\Omega)$ with respect to this norm. 
Table 3

Unified norm in the particular cases of Stokes', Maxwell's and Darcy's problems.

\begin{tabular}{ll}
\hline & $\|[\boldsymbol{v}, q]\|_{X}$ \\
\hline Stokes, $\ell_{S}=L_{0}$ & $v^{\frac{1}{2}}\|\nabla \boldsymbol{v}\|+\frac{v^{\frac{1}{2}}}{L_{0}}\|\boldsymbol{v}\|+v^{-\frac{1}{2}}\|q\|+v^{\frac{1}{2}}\|\nabla \cdot \boldsymbol{v}\|+L_{0} v^{-\frac{1}{2}}\|\nabla q\|_{H^{-1}}$ \\
Maxwell, curl: $\ell_{M}=0$ & $\lambda^{\frac{1}{2}}\|\nabla \times \boldsymbol{v}\|+\frac{\lambda^{\frac{1}{2}}}{L_{0}}\|\boldsymbol{v}\|+\lambda^{-\frac{1}{2}}\|q\|+\lambda^{-\frac{1}{2}} L_{0}\|\nabla q\|$ \\
Maxwell, curl-div: $\ell_{M}=L_{0}$ & $\lambda^{\frac{1}{2}}\|\nabla \times \boldsymbol{v}\|+\frac{\lambda^{\frac{1}{2}}}{L_{0}}\|\boldsymbol{v}\|+\lambda^{-\frac{1}{2}}\|q\|+\lambda^{\frac{1}{2}}\|\nabla \cdot \boldsymbol{v}\|+\lambda^{-\frac{1}{2}} L_{0}\|\nabla q\|_{H^{-1}}$ \\
Darcy, primal: $\ell_{D}=0$ & $\sigma^{\frac{1}{2}}\|\boldsymbol{v}\|+\frac{\sigma^{-\frac{1}{2}}}{L_{0}}\|q\|+\sigma^{-\frac{1}{2}}\|\nabla q\|$ \\
Darcy, dual: $\ell_{D}=L_{0}$ & $\sigma^{\frac{1}{2}}\|\boldsymbol{v}\|+\frac{\sigma^{-\frac{1}{2}}}{L_{0}}\|q\|+\sigma^{\frac{1}{2}} L_{0}\|\nabla \cdot \boldsymbol{v}\|+\sigma^{-\frac{1}{2}}\|\nabla q\|_{H^{-1}}$ \\
\hline
\end{tabular}

Let us discuss how (41) behaves in particular cases. From (39) it is seen that when $v>0$, (39) behaves as the $H^{1}(\Omega)$ norm and (40) as the $H^{-1}(\Omega)$-norm for $\nabla q$, the regularity we may require for $\nabla q$ in the Stokes problem. Suppose that $\nu=0$ and that $\sigma=0$. If $\ell_{M}=0$ then (39) is the $H(\mathbf{c u r l} ; \Omega)$-norm of $\boldsymbol{v}$. From the inf-sup condition between $H(\mathbf{c u r l} ; \Omega)$ and $H_{0}^{1}(\Omega)$ (see e.g. [24]) it follows from (40) that $\|q\|_{Q}$ is equivalent to the $H_{0}^{1}(\Omega)$-norm of $q$. This allows us to conclude that $\ell_{M}=0$ corresponds to the $H(\mathbf{c u r l} ; \Omega) \times H^{1}(\Omega)$ formulation of Maxwell's problem. Analogously, it is easy to see that $\ell_{M}=L_{0}$ corresponds to the $H(\operatorname{curl} ; \Omega) \cap H(\operatorname{div} ; \Omega) \times L^{2}(\Omega)$ formulation. A similar discussion leads to conclude that, in the Darcy case $(\nu=0, \lambda=0), \ell_{D}=0$ corresponds to the mixed primal problem and $\ell_{D}=L_{0}$ to the mixed dual problem.

The limiting cases provided by the norm (41) are summarized in Table 3. The norms of the particular cases have some redundant terms, that are nevertheless required to encompass all possible situations. The objective of a unified functional framework for the combined problem being analyzed is thus accomplished.

Let us proof that problem (38) is stable in the norm (41), stability being expressed in the form of an inf-sup condition, as usual. We define $\operatorname{Ker} B$ as the set of functions that belong to the functional space $V$ such that $\langle\nabla q, \boldsymbol{v}\rangle=0$ for any $q \in Q$. Finally, $X=V \times Q$ and $X_{0}=\operatorname{Ker} B \times Q$.

Theorem 6. The following inf-sup condition holds:

$$
\inf _{[\mathbf{u}, p] \in X_{0} \backslash\{\mathbf{0}, 0\}} \sup _{[\mathbf{v}, q] \in X \backslash\{\mathbf{0}, 0\}} \frac{B_{\mathrm{SMD}}([\boldsymbol{u}, p],[\boldsymbol{v}, q])}{\|[\boldsymbol{u}, p]\|_{X}\|[\boldsymbol{v}, q]\|_{X}} \geqslant C,
$$

with $C>0$ a positive constant.

Proof. First, let us take $[\boldsymbol{v}, q]=[\boldsymbol{u}, p] \in X_{0}$ in the combined problem. We easily obtain:

$$
B_{\mathrm{SMD}}([\boldsymbol{u}, p],[\boldsymbol{u}, p]) \gtrsim\|\boldsymbol{u}\|_{V}^{2},
$$

where the divergence stability terms come from the fact that $\boldsymbol{u}$ is solenoidal. On the other hand, by the definition of the norm $\|\cdot\|_{Q}$, there always exists a function $\boldsymbol{v}_{p} \in V$ such that $\left\|\boldsymbol{v}_{p}\right\|_{V}=\|p\|_{Q}$ and $\left\langle\nabla p, \boldsymbol{v}_{p}\right\rangle=\|p\|_{Q}^{2}$. So, we have that:

$$
B_{\mathrm{SMD}}\left([\boldsymbol{u}, p],\left[\boldsymbol{u}+\alpha \boldsymbol{v}_{p}, p\right]\right) \gtrsim\|\boldsymbol{u}\|_{V}^{2}+\alpha\|p\|_{Q}^{2}+a_{\mathrm{SMD}}\left(\boldsymbol{u}, \alpha \boldsymbol{v}_{p}\right),
$$

where $a_{\mathrm{SMD}}(\boldsymbol{u}, \boldsymbol{v})=v(\nabla \boldsymbol{u}, \nabla \boldsymbol{v})+\lambda(\nabla \times \boldsymbol{u}, \nabla \times \boldsymbol{v})+\sigma(\boldsymbol{u}, \boldsymbol{v})$. Now, noting that $a_{\mathrm{SMD}}$ is continuous with respect to $\|\cdot\|_{V}$, we can prove the theorem taking $\alpha>0$ small enough in the previous expression together with Cauchy-Schwarz and Young's inequalities.

The previous inf-sup condition, in $\operatorname{Ker} B$, is enough to prove the well-posedness of the problem (see, e.g., [16, Theorem 2.34.]).

\subsection{Stabilized finite element approximation}

The stabilized finite element formulation of problem (38) we propose is a generalization of the one presented for the three independent model problems, keeping in mind the reformulation of the approximation of Maxwell's problem as a residual based stabilized finite element method.

If $V_{h} \times Q_{h}$ is a finite element space to approximate $V \times Q$, the discrete problem reads: find $\left[\boldsymbol{u}_{h}, p_{h}\right] \in V_{h} \times Q_{h}$ such that

$$
B_{\mathrm{SMD}, h}([\boldsymbol{u}, p],[\boldsymbol{v}, q])=L_{\mathrm{SMD}, h}([\boldsymbol{v}, q]),
$$

for all $\left[\boldsymbol{v}_{h}, q_{h}\right] \in V_{h} \times Q_{h}$, where $B_{S M D} h$ and $L_{S M D, h}$ are the forms in the stabilized finite element formulation. Stokes' and Darcy's problems allow one the use of ASGS, OSS and SSW techniques. However, Maxwell's problem only allows the SSW formulation or an OSS formulation with a weakened stability. For this reason, when considering a unified treatment of all the problems at hand, we only use the SSW and OSS methods. 
Skew symmetric weighting method. The forms for the unified Stokes-Maxwell-Darcy problem approximated via an SSW finite element discretization read as follows:

$$
\begin{aligned}
B_{\mathrm{SMD}, h}\left(\left[\boldsymbol{u}_{h}, p_{h}\right],\left[\boldsymbol{v}_{h}, q_{h}\right]\right)= & B_{\mathrm{SMD}}\left(\left[\boldsymbol{u}_{h}, p_{h}\right],\left[\boldsymbol{v}_{h}, q_{h}\right]\right)+\tau_{p} \sum_{K}\left\langle\nabla \cdot \boldsymbol{u}_{h}, \nabla \cdot \boldsymbol{v}_{h}\right\rangle_{K} \\
& +\tau_{u} \sum_{K}\left\langle-v \Delta \boldsymbol{u}_{h}+\sigma \boldsymbol{u}_{h}+\nabla p_{h}, \nabla q_{h}\right\rangle_{K}, \\
L_{S, h}\left(\left[\boldsymbol{v}_{h}, q_{h}\right]\right)=\left(\boldsymbol{f}, \boldsymbol{v}_{h}\right)+ & \tau_{u} \sum_{K}\left\langle\boldsymbol{f}, \nabla q_{h}\right\rangle_{K}+\left(g, q_{h}\right)+\tau_{p} \sum_{K}\left\langle g, \nabla \cdot \boldsymbol{v}_{h}\right\rangle_{K} .
\end{aligned}
$$

Let us stress the fact that the curl-curl term in the stabilization term weighted with $\tau_{u}$ has been canceled out, as commented above for the Maxwell problem. A critical issue is the calculation of the stabilization parameters $\tau_{p}, \tau_{u}$, which are the same in (43) and in (49) below. They have to behave as the parameters of the model problems when the appropriate physical parameters tend to zero. In the case of Maxwell's and Darcy's problems, they also have to allow one to encompass the two functional settings we have discussed for each of these problems. It is immediately checked that both goals are achieved if we take

$$
\tau_{p}=c_{1} v+c_{2} \lambda \frac{\ell_{M, h}^{2}}{L_{0}^{2}}+c_{3} \sigma \ell_{D, h}^{2}, \quad \tau_{u}=\frac{h^{2}}{\tau_{p}} .
$$

The values of $\ell_{M, h}$ and $\ell_{D, h}$ may be either $h$ or $L_{0}$, corresponding to the values of $\ell_{M}$ and $\ell_{D}$ of 0 and $L_{0}$, respectively. Note that, for stability reasons, $\ell_{M, h}$ and $\ell_{D, h}$ cannot be 0 .

For the SSW formulation in (43), the stability and error analysis can be undertaken in the mesh dependent norm:

$$
\left\|\left[\boldsymbol{v}_{h}, q_{h}\right]\right\|_{X, h}^{2}:=L_{0}^{-2} \kappa\left\|\boldsymbol{v}_{h}\right\|^{2}+v\left\|\nabla \boldsymbol{v}_{h}\right\|^{2}+\lambda\left\|\nabla \times \boldsymbol{v}_{h}\right\|^{2}+\tau_{p}\left\|\nabla \cdot \boldsymbol{u}_{h}\right\|^{2}+\tau_{u} \sum_{K}\left\|\nabla q_{h}\right\|_{K}^{2} .
$$

From this norm it is readily seen that we have all the control expected. In particular, in the applications the most interesting situations are the curl formulation for the Maxwell problem and the dual formulation for the Darcy problem. If we take $\ell_{M, h}=L_{0}$ and $\ell_{D, h}=h$, these will be precisely the limiting cases when $v \rightarrow 0, \sigma \rightarrow 0$ and $\nu \rightarrow 0, \lambda \rightarrow 0$, respectively.

The following theorem proves that the SSW is stable. To avoid technicalities, we present this stability result in the form of a bound for the unknowns in terms of the data rather than as an inf-sup condition.

Theorem 7. The solution $\left[\boldsymbol{u}_{h}, p_{h}\right]$ of problem (42) satisfies the stability bound:

$$
\left\|\left[\boldsymbol{u}_{h}, p_{h}\right]\right\|_{X, h} \lesssim C_{f, g},
$$

where $C_{f, g}$ is used to denote a generic constant that depends on the norm of the data (but is independent of $h$ ). Moreover

$$
\left\|p_{h}\right\|_{Q} \lesssim C_{f, g} .
$$

Proof. The stabilized problem is coercive for the whole mesh-dependent norm, except for the $\lambda\left\|\boldsymbol{u}_{h}\right\|^{2}$ term, and therefore all terms in $\left\|\left[\boldsymbol{u}_{h}, p_{h}\right]\right\|_{X, h}$ except this one will be bounded by the appropriate norm of the data. For $\ell_{M, h}=L_{0}$, this result is straightforward from the fact that

$$
L_{0}^{-1}\left\|\boldsymbol{u}_{h}\right\| \lesssim\left\|\nabla \times \boldsymbol{u}_{h}\right\|+\left\|\nabla \cdot \boldsymbol{u}_{h}\right\| .
$$

For $\ell_{M, h}=h$, this term can still be bounded by using the technical result in [5, Lemma 3], that gives:

$$
L_{0}^{-2} \lambda\left\|\boldsymbol{u}_{h}\right\|^{2} \lesssim \lambda\left\|\nabla \times \boldsymbol{u}_{h}\right\|^{2}+\lambda \frac{h^{2}}{L_{0}^{2}}\left\|\nabla \cdot \boldsymbol{u}_{h}\right\|^{2}+L_{0}^{2} \lambda^{-1}\left\|p_{h}\right\|^{2} .
$$

Then, multiplying the previous inequality against

$$
\tau_{u} \lambda L_{0}^{-2}=\frac{\lambda \frac{h^{2}}{L_{0}^{2}}}{c_{1} v+c_{2} \lambda \frac{h^{2}}{L_{0}^{2}}+c_{3} \sigma \ell_{D, h}^{2}} \lesssim 1
$$

we get

$$
L_{0}^{-2} \lambda\left\|\boldsymbol{u}_{h}\right\|^{2} \frac{\lambda \frac{h^{2}}{L_{0}^{2}}}{c_{1} v+c_{2} \lambda \frac{h^{2}}{L_{0}^{2}}+c_{3} \sigma \ell_{D, h}^{2}} \lesssim \lambda\left\|\nabla \times \boldsymbol{u}_{h}\right\|^{2}+\tau_{p}\left\|\nabla \cdot \boldsymbol{u}_{h}\right\|^{2}+\tau_{u}\left\|p_{h}\right\|^{2},
$$


where the right-hand side is bounded in terms of the data. Now, we use the already known stability bounds over $L_{0}^{-2}\left(\sigma L_{0}^{2}+\right.$ v) $\left\|\boldsymbol{u}_{h}\right\|^{2}$. We have that:

$$
L_{0}^{-2} \lambda\left\|\boldsymbol{u}_{h}\right\|^{2} \frac{\lambda \frac{h^{2}}{L_{0}^{2}}}{c_{1} v+c_{2} \lambda \frac{h^{2}}{L_{0}^{2}}+c_{3} \sigma \ell_{D, h}^{2}}+L_{0}^{-2}\left(\sigma \ell_{D, h}^{2}+v\right)\left\|\boldsymbol{u}_{h}\right\|^{2} \leqslant C_{f, g} .
$$

Using the relation

$$
a<\frac{a^{2}}{a+b}+b
$$

for any $a, b>0$ in the previous equation, we easily get the bound over $L_{0}^{-1} \lambda\left\|\boldsymbol{u}_{h}\right\|^{2}$.

With regard to the pressure, we can always find a $\boldsymbol{v}_{p} \in X$ such that

$$
\left\|p_{h}\right\|_{Q}^{2}=\left\langle\nabla p_{h}, \boldsymbol{v}_{p}\right\rangle
$$

and $\left\|\boldsymbol{v}_{p}\right\|_{V}=\left\|p_{h}\right\|_{Q}$, by the definition of the norm. On the other hand, let us consider an optimal projection of $\boldsymbol{v}_{p}$ onto the finite element space, denoted by $\tilde{\boldsymbol{v}}_{p, h}$ (see, e.g., [6]), e.g. the Scott-Zhang projection. Invoking the problem solved by the solution $\left[\boldsymbol{u}_{h}, p_{h}\right]$, we have

$$
\left\|p_{h}\right\|_{Q}^{2}=\left\langle\nabla p_{h}, \boldsymbol{v}_{p}-\tilde{\boldsymbol{v}}_{p, h}\right\rangle-a_{\mathrm{SMD}}\left(\boldsymbol{u}_{h}, \tilde{\boldsymbol{v}}_{p, h}\right)-\tau_{p} \sum_{K}\left\langle\nabla \cdot \boldsymbol{u}_{h}, \nabla \cdot \tilde{\boldsymbol{v}}_{p, h}\right\rangle_{K}+\left(\boldsymbol{f}, \tilde{\boldsymbol{v}}_{p, h}\right) .
$$

The first term is bounded as follows:

$$
\begin{aligned}
\left\langle\nabla p_{h}, \boldsymbol{v}_{p}-\tilde{\boldsymbol{v}}_{p, h}\right\rangle & \lesssim\left(\frac{h^{2}}{v+\lambda h^{2} L_{0}^{-2}+\sigma h^{2}}\right)^{\frac{1}{2}}\left\|\nabla p_{h}\right\|\left(\frac{h^{2}}{v+\lambda h^{2} L_{0}^{-2}+\sigma h^{2}}\right)^{-\frac{1}{2}}\left\|\boldsymbol{v}_{p}-\tilde{\boldsymbol{v}}_{p, h}\right\| \\
& \lesssim\left(\frac{h^{2}}{v+\lambda h^{2} L_{0}^{-2}+\sigma h^{2}}\right)^{\frac{1}{2}}\left\|\nabla p_{h}\right\|\left\|\boldsymbol{v}_{p}\right\|_{V},
\end{aligned}
$$

where we have used the interpolation properties and stability of the Scott-Zhang projector. The rest of the terms in (47) are easily bounded in terms of the data using the bounds already proved for $\left\|\left[\boldsymbol{u}_{h}, p_{h}\right]\right\|_{X, h}$.

Orthogonal subscales method. For the combined problem we can also consider the following unified formulation:

$$
\begin{aligned}
& B_{\mathrm{SMD}, h}\left(\left[\boldsymbol{u}_{h}, p_{h}\right],\left[\boldsymbol{v}_{h}, q_{h}\right]\right)=B_{\mathrm{SMD}}\left(\left[\boldsymbol{u}_{h}, p_{h}\right],\left[\boldsymbol{v}_{h}, q_{h}\right]\right)+\tau_{p} \sum_{K}\left\langle P_{h}^{\perp}\left(\nabla \cdot \boldsymbol{u}_{h}\right), P_{h}^{\perp}\left(\nabla \cdot \boldsymbol{v}_{h}\right)\right\rangle_{K} \\
& +\tau_{u} \sum_{K}\left\langle P_{h}^{\perp}\left(\nabla p_{h}\right), P_{h}^{\perp}\left(\nabla q_{h}\right)\right\rangle_{K}, \\
& L_{S, h}\left(\left[\boldsymbol{v}_{h}, q_{h}\right]\right)=\left(\boldsymbol{f}, \boldsymbol{v}_{h}\right)+\left(g, q_{h}\right),
\end{aligned}
$$

when using the OSS formulation. However, in this case the norm (44) has to be weakened for the OSS method (49) in what concerns control on the pressure gradient projected onto the finite element space, as explained at the end of Section 2.4.2.

We omit the details of the convergence analysis for the formulations presented, which follow along the same lines as those of the individual problems.

\section{Conclusions}

The purpose of this paper has been twofold. On the one side, we have summarized in a unified manner the formulation and the analysis we had presented in [4,3,5]. We have shown that the stabilized formulations we propose are able not only to allow arbitrary interpolations of $\boldsymbol{u}$ and $p$, but also to resort to the appropriate functional setting of the problem with a proper design of the stabilization parameters (curl or curl-div formulations for Maxwell's problem, primal or dual formulations for Darcy's problem). Stability and optimal convergence results in fully meaningful norms have been presented.

On the other hand, we have also proposed a formulation for a combined problem able to reduce correctly to the model problems when the physical parameters tend to zero. A stabilized formulation has also been introduced for this unified problem. Let us just remark the difficulty encountered to formulate this method as residual based because of the lack of control of the double curl of $\boldsymbol{u}$ if $\ell_{M, h}=L_{0}$, the case of interest if singular solutions need to be approximated. 


\section{References}

[1] D.N. Arnold, P.B. Bochev, R.B. Lehoucq, R.A. Nicolaides, M. Shashkov (Eds.), Compatible Spatial Discretizations, The IMA Volumes in Mathematics and its Applications, Springer, 2006.

[2] D.N. Arnold, R.S. Falk, R. Winther, Finite element exterior calculus, homological techniques, and applications, Acta Numerica 15 (2006) 1-155.

[3] S. Badia, R. Codina, Unified stabilized finite element formulations for the Stokes and the Darcy problems, SIAM Journal on Numerical Analysis 47 (3) (2009) 1977-2000.

[4] S. Badia, R. Codina, Stabilized continuous and discontinuous Galerkin techniques for Darcy flow, Computer Methods in Applied Mechanics and Engineering 199 (2010) 1654-1667.

[5] S. Badia, R. Codina, A nodal-based finite element approximation of the Maxwell problem suitable for singular solutions, submitted for publication.

[6] S.C. Brenner, L.R. Scott, The Mathematical Theory of Finite Element Methods, Springer-Verlag, 1994.

[7] S.C. Brenner, F. Li, L.-Y. Sung, A locally divergence-free interior penalty method for two-dimensional curl-curl problems, SIAM Journal on Numerical Analysis 46 (2008) 1190-1211.

[8] F. Brezzi, M. Fortin, Mixed and Hybrid Finite Element Methods, Springer-Verlag, 1991.

[9] A.N. Brooks, T.J.R. Hughes, Streamline upwind/Petrov-Galerkin formulations for convection dominated flows with particular emphasis on the incompressible Navier-Stokes equation, Computer Methods in Applied Mechanics and Engineering 32 (1982) 199-259.

[10] R. Codina, Analysis of a stabilized finite element approximation of the Oseen equations using orthogonal subscales, Applied Numerical Mathematics 58 (2008) 264-283.

[11] R. Codina, J. Blasco, A finite element formulation for the Stokes problem allowing equal velocity-pressure interpolation, Computer Methods in Applied Mechanics and Engineering 143 (1997) 373-391.

[12] R. Codina, J. Blasco, G.C. Buscaglia, A. Huerta, Implementation of a stabilized finite element formulation for the incompressible Navier-Stokes equations based on a pressure gradient projection, International Journal for Numerical Methods in Fluids 37 (2001) 419-444.

[13] M. Costabel, A coercive bilinear form for Maxwell's equations, Journal of Mathematical Analysis and Applications 157 (2) (1991) $527-541$.

[14] M. Costabel, M. Dauge, Weighted regularization of Maxwell equations in polyhedral domains, Numerische Mathematik 93 (2) (2002) $239-277$.

[15] M. Crouzeix, P.A. Raviart, Conforming and nonconforming finite element methods for solving the stationary Stokes equations, RAIRO Anal. Numer. 3 (1973) 33-75.

[16] A. Ern, J.L. Guermond, Theory and Practice of Finite Elements, Springer-Verlag, 2004.

[17] C. Hazard, Numerical simulation of corner singularities: a paradox in Maxwell-like problems, Comptes Rendus-Mécanique 330 (1) (2002) 57-68.

[18] T.J.R. Hughes, Multiscale phenomena: Green's function, the Dirichlet-to-Neumann formulation, subgrid scale models, bubbles and the origins of stabilized formulations, Computer Methods in Applied Mechanics and Engineering 127 (1995) 387-401.

[19] T.J.R. Hughes, L.P. Franca, M. Balestra, A new finite element formulation for computational fluid dynamics: V. Circumventing the Babuŝka-Brezzi condition: a stable Petrov-Galerkin formulation for the Stokes problem accommodating equal-order interpolations, Computer Methods in Applied Mechanics and Engineering 59 (1986) 85-99.

[20] A. Masud, T.J.R. Hughes, A stabilized mixed finite element method for Darcy flow, Computer Methods in Applied Mechanics and Engineering 191 (2002) 4341-4370.

[21] J.C. Nedelec, Mixed finite elements in $\mathbb{R}^{3}$, Numer. Meth. 35 (1980) 119-136

[22] J.C. Nedelec, A new family of mixed finite elements in $\mathbb{R}^{3}$, Numer. Meth. 50 (1986) 57-81.

[23] P.A. Raviart, J.M. Thomas, A mixed-finite element method for second order elliptic problems, in: Mathematical Aspects of the Finite Element Method, in: Lecture Notes in Mathematics, Springer, New York, 1977.

[24] D. Schötzau, Mixed finite element methods for stationary incompressible magneto-hydro-dynamics, Numer. Meth. 96 (2004) 771-800.

[25] T. Sorokina, A.J. Worsey, A multivariate Powell-Sabin interpolant, Advances in Computational Mathematics 29 (2008) 71-89. 